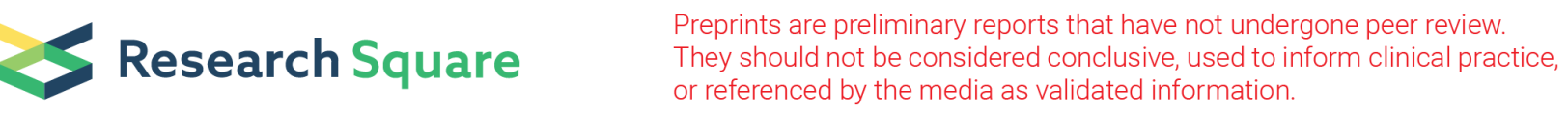

\title{
Periodontal disease status and treatment need among a Kurdish population in Sulaimani/ Iraq as assessed by CPITN index.
}

Sarhang Gul ( $\sim$ sarhang.hama@univsul.edu.iq)

Research article

Keywords: Periodontal diseases, Epidemiology, CPITN, Kurdish population

Posted Date: September 10th, 2019

DOI: https://doi.org/10.21203/rs.2.14245/v1

License: (c) (i) This work is licensed under a Creative Commons Attribution 4.0 International License.

Read Full License 


\section{Abstract}

Background This study identifies the prevalence and severity of periodontal diseases in a given population of Sulaimani using the Community Periodontal Index of Treatment Needs (CPITN) and attempts to find the relation of CPITN with age, sex, location, visit to dentist, tooth brushing, use of mouth wash and interdental aids, systemic disease, smoking status and missing teeth.

Methods Subjects attending Periodontics clinics at College of Dentistry, University of Sulaimani were examined. Periodontal examinations were performed using CPITN, plaque index (PI) and bleeding index (BI). Other data including location, visit to dentist, tooth brushing, use of mouth wash and interdental aids, presence of systemic diseases, smoking status and missing teeth were also collected. The data were subjected to logistic regression to find a relation between periodontal status (CPITN $\geq 3$ or not as dependent variable) and other variables.

Results Among the 1354 subjects (mean age $30.8 \pm 12.9$ years) examined, $43.6 \%$ were male and the majority of these (80.9\%) were from urban areas. The mean PI and BI were $0.64 \pm 24$ and $0.39 \pm 0.28$, respectively. Calculus was the most prevalent highest score amongst examined subjects $(62.2 \%)$, followed by shallow pocket $(22.2 \%)$ and bleeding (9\%). Among the sextants, lower central sextants were found to have the highest percentage of calculus (73.1\%), followed by lower left (49.7\%) and lower right (48.7\%). The highest percentages of shallow and deep pockets were found at upper left $(14 \%, 2.5 \%$, respectively) and upper right $(11.4 \%, 3.2 \%$, respectively) sextants. Logistic regression analysis showed that age, sex, frequency of brushing and smoking significantly correlated with CPITN scores.

Conclusion Prevalence and severity of periodontal diseases are high in the examined population and at population level age, sex, brushing and smoking were shown to have an impact on periodontal condition.

\section{Background}

Periodontal diseases are a group of diseases that affect supporting structures of the teeth and are considered as a main cause of tooth loss in adults worldwide. Although they share similar aetiologies, different natural courses, prognoses and responses to conventional therapy have been reported (1). The Community Periodontal Index of Treatment Needs (CPITN) is supported by both the World Dental Federation (FDI) and World Health Organization (WHO) as epidemiological screening criteria to determine periodontal treatment needs in a population. The CPITN has been used widely to plan for health services; furthermore, the data retrieved from it provide a base to determine the needs of the overall population in terms of the requirements for specific types of treatment and the clinical personnel involved in periodontal care (2).

A series of global epidemiological studies of periodontal disease have indicated that probing pocket depth (PPD) and clinical attachment loss improved in the United Kingdom and the United States between 1988 and 2004. However, the prevalence of periodontal disease increased in other countries over the same period. These countries include Germany and Hungary, in which the prevalence of scores 3 and 4 
increased among adults aged 35-44 and 65-74 years from 1997 to $2005(3-5)$. These figures indicate implementation of efficacious and low-cost periodontal healthcare is urgently needed in many parts of the world.

Tooth cleanliness, as measured by plaque and calculus accumulation, plays an essential role in periodontal health (6). In adults with periodontal disease, oral health-related quality of life can be moderately improved through basic periodontal treatments (7) including routine non-surgical therapies and removal of dental biofilms and biofilm-retentive calculus by mechanical means (i.e., scaling and root surface debridement) (8).

Since the 1940s, there has been great general interest among epidemiologists in development of a suitable index system for quantification of the prevalence and severity of periodontal diseases in individuals as well as in population groups in all parts of the world (9). The CPITN was jointly developed by the WHO and FDI (10) and since then it has been used worldwide to study periodontal disease in populations at community level, as well as the related treatment needs. It is claimed to be a simple, rapid, inexpensive, easily applied index which requires minimum equipment and has an international uniformity (11). However, baseline data on periodontal knowledge, prevalence and severity of periodontal disease in a Kurdish population in Northern Iraq are not yet available, either from the WHO or individual utilization of this or any other periodontal disease index. Therefore, the aims of this study are to determine periodontal health status in a Kurdish population in Sulaimani/Iraq and find the impact of age, sex, location, visit to dentist, tooth brushing, use of mouth wash and interdental aids, systemic disease and smoking status on periodontal condition among the population base.

\section{Methods}

This cross-sectional study was conducted on patients attending the Periodontics Department of the College of Dentistry, University of Sulaimani from September 2016 to June 2018. The study protocol was approved by the ethical committee of the Faculty of Medicine, University of Sulaimani (ethical approval number: 342). All participants signed an informed consent form after receiving a detailed information sheet about the study. Inclusion criteria were: patients aged $\geq 18$ years old, with two or more teeth present in each sextant and not indicated for extraction. A single expert examiner collected all clinical parameters with $98 \%$ agreement of intra-examiner calibration.

The data were obtained through a data collection sheet that recorded the following: age (age group <20, $20-29,30-39,40-49,50-59$ and $\geq 60$ ), sex, location (urban and rural), visit to dentist (No, regular and irregular), tooth brushing (no brushing or how many times per day), use of interdental hygiene (yes or no) and type (dental floss, interdental brush, single tufted brush), systemic disease (such as diabetes, hypertension), smoking status (yes or no) and if yes: how many cigarettes per day and missing teeth. Periodontal condition was assessed by CPITN index, bleeding index (BI) and plaque index (PI) of all teeth using WHO probe (12) (Figure 1). The CPITN is a practical index for population-based surveys. It is used as an indicator of periodontal status in a specific community or population (13). The CPITN measures the 
following conditions: no treatment needs (score 0), bleeding gingiva on gentle probing (score 1), presence of supra or subgingival calculus or other plaque retentive factors (score 2), 4 to $5 \mathrm{~mm}$ PPD (score 3 ), and $\geq 6 \mathrm{~mm}$ PPD (score 4) (14). The dentition was divided into sextants and the highest scores in terms of the worst periodontal condition around each tooth in that sextant were recorded.

\section{Statistical analysis}

Advice was taken from an expert statistician. Continuous data (age, missing teeth, $\mathrm{PI}$ and $\mathrm{BI}$ ) were subjected first to the Shapiro-Wilk test for normal distribution and thereafter to appropriate parametric or nonparametric tests. A single overall CPITN score, calculated as the maximum score found over each sextant, was assigned to each sextant and the highest score in all sextants was assigned to each subject. Periodontal condition was then presented as percentage per given population. The highest CPITN score per subject was used an outcome dependent variable dichotomized using accepted thresholds (15) of low $($ CPITN $<3)$ and high (CPITN $\geq 3$ ) for multiple logistic regression modeling. The association between periodontal condition and other confounders such as age, sex, location, visit to dentist, tooth brushing, use of interdental hygiene, systemic disease and smoking status and missing teeth was determined by logistic regression using the highest CPITN score per subject. Statistical significance was defined as $p \leq$ 0.05. All calculations were performed using the SPSS software package (version 21; SPSS Inc., Chicago, IL, USA).

\section{Results}

During the study 1354 subjects ( $43.6 \%$ male and $56.4 \%$ female) with mean age of 30.812 .9 years were examined. The median of missing teeth in the examined population is 4 teeth (inter quartile range 2-8 teeth) with mean PI of 0.6424 and mean BI of 0.390 .28 (Figure 2). The majority of subjects were from urban areas (80.9\%) and visited the dentist irregularly (81.3\%) (Table 1). Just above half of the examined subjects brushed their teeth once a day and $67.1 \%$ did not use interdental aids, while mouth wash was used by only $8.3 \%$ (Table 1). Systemic diseases such as hypertension and diabetes were detected in only $5.7 \%$ and $4.2 \%$ of the examined population, respectively, and $79.2 \%$ were non-smokers (Table 1 ).

At population level, calculus (score 2 ) was the most prevalent periodontal condition (62.2\%), followed by shallow pocket (score 3 ) with $22.2 \%$. Bleeding (score 1 ) and deep periodontal pocket (score 4) were detected at $9 \%$ and $6.1 \%$, respectively (Figure 3). At sextant level, calculus (score 2 ) was mostly detected at the lower anterior sextant (73.1\%), whereas shallow pocket (score 3 ) and deep pocket (score 4) were most prevalent on the upper right (11.4\% and 3.2\%, respectively) and left ( $14 \%$ and $2.5 \%$, respectively) sextants. Bleeding (score 1) was most prevalent at the upper anterior sextant (54.5\%) (Figure 4). Furthermore, at age group level, the percentage of subjects with shallow and deep pocket increased steadily with age. For example, only $0.8 \%$ of subjects aged $<20$ years old had deep pocket, but this figure increased to $2.9 \%, 7 \%, 8.9 \%, 18.6 \%$ and $23.4 \%$ for the age groups $20-29,30-39,40-49,50-59$ and $>60$ years, respectively. In contrast, periodontal conditions determined with score 1 and 2 declined gradually with increasing age (Table 2). 
Logistic regression was used to determine the relations between the examined variables and periodontal condition and it was revealed that age, sex (male), brushing and smoking are statistically significantly associated with poor periodontal condition (Table 3). Amongst those variables, age exhibited the highest odd ratio $(6.1,95 \% \mathrm{Cl}$ of $1.4-12.2)$. Meanwhile, other variables such as living area, visit to dentist, use of interdental aids and mouth wash plus presence of diabetes and hypertension showed no statistically significant association with periodontal condition at population level (Table 3).

\section{Discussion}

This study has employed CPITN to investigate the prevalence of periodontal diseases in the Kurdish population of Sulaimani; additionally, the impacts on periodontal condition of other confounders such as age, sex, location, visit to dentist, tooth brushing, use of mouth wash and interdental aids, systemic disease, smoking status and missing teeth were examined. It is important to acknowledge that prevalence of periodontal diseases amongst the Kurdish population had not previously been examined using any criteria for determining periodontal diseases. As a first attempt to determine periodontal disease in this population, this study employed CPITN as it is extensively used in epidemiological studies for periodontal diseases and would therefore allow comparative assessment of the data of this study. Although CPITN has been criticized for inaccuracy in gingivitis and calculus detection and for generally underestimating periodontitis, this index is still widely accepted as a simple, quick to implement and reproducible assessment tool (16).

Among the subjects examined, calculus (score 2) was the most common highest score (62.2\%), which is in line with other studies $(3,17,18)$. Severe periodontitis, as represented by score 4 , was found in only $6.1 \%$ of the examined population (Figure 3), which is in accordance with the data reported in Hungary (19) and Norway (20). Whereas, Pilot \& Miyazaki (21) in a European population found higher (10\% to $15 \%)$ prevalence of periodontitis. These differences can be explained by the fact that periodontal diseases are multifactorial diseases, meaning that factors such as genetics, medication, systemic diseases, habit (such as smoking) and frequency of tooth brushing have an impact on the extent and severity of this disease and these confounders vary from one place to another.

At sextant level, calculus is most prevalent at lower anterior sextant, which is obviously related to the presence of the Wharton's duct, and this is in agreement with other studies $(22,23)$. Meanwhile, both shallow and deep pockets are most prevalent at upper posterior sextants and this is again similar to the result of other research (23) (Figure 4).

Logistic regression showed that only age, sex (male), brushing and smoking status have impact on periodontal condition $(P<0.05$, Table 3$)$. Periodontal condition was poorer in males than females and this is similar to the data reported by other studies $(19,24,25)$. Age is another factor found to have impact on periodontal condition, with the present study showing a steady increase in prevalence and severity of periodontal disease with age (Table 2) which is in accordance with the WHO data bank and results of other studies $(26,27)$. Smoking is well-known as a risk factor that contributes to poor periodontal 
condition, and the present study found both current and former smoking to be associated with poor periodontal condition (odd ratio $=1.8$ and 1.4 , respectively), which is in accordance with previous studies $(28,29)$. Brushing is another factor shown to have impact on periodontal condition (odd ratio $=2.1$ ), while at population level, other factors such as interdental aids, mouth wash, residence place, visit to dentist frequency and systemic diseases such diabetes and hypertension showed no effect on periodontal condition, which is not in agreement with other studies $(28,30,31)$. This can be related to factors such as small sample size and the limitations of the CPITN index mentioned earlier.

\section{Conclusion}

Prevalence and severity of periodontal diseases are high in the examined population and age, sex, brushing and smoking were found to have an impact on periodontal condition at population level. Further studies with larger sample size and full mouth examination are recommended. Furthermore, in Sulaimani, this study shows that periodontal condition and oral hygiene standards need improvement. Therefore, to prevent and control periodontal disease, a comprehensive intervention program is recommended at regional level.

\section{Declarations}

Acknowledgment and disclosure

The author thanks Asst. Prof. Dr. Faraedon Zardawi and Dr. Dler Ali Khurshed for their help with the study. The author has no conflict of interest to declare.

\section{References}

1. Page RC, Beck JD. Risk assessment for periodontal diseases. Int Dent J.1997;47(2):61-87.

2. Cutress TW, Ainamo J, Sardo-Infirri J. The community periodontal index of treatment needs (CPITN) procedure for population groups and individuals. Int Dent J. 1987;37(4):222-33.

3. Hugoson A, Sjodin B, Norderyd O. Trends over 30 years, 1973-2003, in the prevalence and severity of periodontal disease. J Clin Periodontol. 2008;35(5):405-14.

4. Holtfreter B, Kocher T, Hoffmann T, Desvarieux M, Micheelis W. Prevalence of periodontal disease and treatment demands based on a German dental survey (DMS IV). J Clin Periodontol. 2010;37(3):211-9. 区

5. Dye BA. Global periodontal disease epidemiology. Periodontol 2000. 2012;58(1):10-25. \

6. Morris AJ, Steele J, White DA. The oral cleanliness and periodontal health of UK adults in $1998 . \mathrm{Br}$ Dent J. 2001;191(4):186-92. 『

7. Shanbhag S, Dahiya M, Croucher R. The impact of periodontal therapy on oral health-related quality of life in adults: a systematic review. J Clin Periodontol. 2012;39(8):725-35. \&

8. Slots J. Low-cost periodontal therapy. Periodontol 2000. 2012;60(1):110-37. 《 
9. Ainamo J, Ainamo A. Validity and relevance of the criteria of the CPITN. Int Dent J. 1994;44(5 Suppl 1):527-32.

10. Ainamo J, Barmes D, Beagrie G, Cutress T, Martin J, Infirri JS. Development of the World Health Organization (WHO) community periodontal index of treatment needs (CPITN). Int Dent J. 1982;32(3):281-91.

11. Pilot T, Miyazaki H. Global results: 15 years of CPITN epidemiology. Int Dent J. 1994;44(5 Suppl 1):553-60.

12. Gul SS, Douglas CW, Griffiths GS, Rawlinson A. A pilot study of active enzyme levels in gingival crevicular fluid of patients with chronic periodontal disease. J Clin Periodontol 2016;43(8):629-36.

13. Ainamo J, Barmes D, Beagrie G, Cutress T, Martin J, Sardoinfirri J. Development of the World-HealthOrganization (WHO) commu- nity periodontal index of treatment needs (CPITN). Int Dent J 1982;32(3):281-91.

14. Benigeri M, Brodeur JM, Payette M, Charbonneau A, Ismail Al. Community periodontal index of treatment needs and prevalence of periodontal conditions. J Clin Periodontol. 2000;27(5):308-12.

15. Angeli F, Verdecchia, P, Pellegrino C, Pellegrino RG, Pellegrino G, Prosciutti L, Giannoni C, Cianetti S, Bentivoglio M. Association between periodontal disease and left ventricle mass in essential hypertension. Hypertension. 2003;41(3)488-92.

16. El-Qaderi SS, Quteish Ta'ani D. Assessment of periodontal knowledge and periodontal status of an adult population in Jordan. Int J Dent Hyg. 2004;2(3):132-6.

17. Bansal M, Mittal N, Singh TB. Assessment of the prevalence of periodontal diseases and treatment needs: A hospital-based study. J Indian Soc Periodontol. 2015;19(2):211-5.

18. Nethravathi TD, Joshipura V, Venugopal S, Subbaiah SK, Jagadeesh KN, Apparaju V. A comparative assessment of periodontal status and treatment needs among population in Tumkur district using CPITN: An epidemiological study. J Adv Clin Res Insi. 2015;2(3):120-3

19. Hermann P1, Gera I, Borbély J, Fejérdy P, Madléna M. Periodontal health of an adult population in Hungary: findings of a national survey. J Clin Periodontol. 2009;36(6):449-57.

20. Skudutyte-Rysstad, R., Eriksen, H. M. \& Hansen, B. F. (2007) Trends in periodontal health among 35year-olds in Oslo, 1973-2003. J Clin Periodontol. 2007:34(10):867-72.

21. Pilot T, Miyazaki H. Periodontal conditions in Europe. J Clin Periodontol. 1991;18(6):353-7.

22. Jin Y, Yip HK. Supragingival calculus: formation and control. Crit Rev Oral Biol Med. 2002;13(5):42641.

23. Kovacević V, Milosavljević M, Rancić N, Daković D. Assessment of the periodontal health and community periodontal index in the Army of Serbia. Vojnosanit Pregl. 2015;72(11):953-60.

24. Oliver RC, Brown LJ, Lo"e H. Periodontal disease in the United States population. J Periodontol. 1998;69(2):269-78.

25. Albander JM, Kingman A. Gingival recession, gingival bleeding, and dental calculus in adults 30 years of age and older in the United States, 1988-1994. J Periodontol. 1999;70(1):30-43. 
26. Petersen PE. The World Oral Health Report 2003: continuous improvement of oral health in the $21 \mathrm{st}$ century -the approach of the WHO Global Oral Health Programme. Community Dent Oral Epidemiol. 2003;31(Suppl 1):3-24.

27. World Health Organization. The WHO Global Oral Health Data Bank. 2003. Geneva: World Health Organization.

28. Cepeda MS, Weinstein R, Blacketer C, Lynch MC. Association of flossing/inter-dental cleaning and periodontitis in adults. J Clin Periodontol. 2017;44(9):866-71.

29. Chaffee BW, Couch ET, Ryder MI. The tobacco-using periodontal patient: role of the dental practitioner in tobacco cessation and periodontal disease management. Periodontol 2000. 2016;71(1):52-64.

30. Sälzer S, Slot DE, Van der Weijden FA, Dörfer CE. Efficacy of inter-dental mechanical plaque control in managing gingivitis-a meta-review. J Clin Periodontol. 2015;42(Suppl 16):S92-105.

31. Sanz M, Ceriello A, Buysschaert M, Chapple I, Demmer RT, Graziani F, Herrera D, Jepsen S, Lione L, Madianos P, Mathur M, Montanya E, Shapira L, Tonetti M, Vegh D. Scientific evidence on the links between periodontal diseases and diabetes: Consensus report and guidelines of the joint workshop on periodontal diseases and diabetes by the International Diabetes Federation and the European Federation of Periodontology. J Clin Periodontol. 2018;45(2):138-49

\section{Tables}

Table 1: Characteristics of examined population.

\begin{tabular}{|c|c|c|c|c|c|}
\hline \multicolumn{2}{|c|}{ Variables } & Percentage & \multicolumn{2}{|c|}{ Variables } & Percentage \\
\hline \multirow[t]{2}{*}{ Sex } & Male & 43.6 & \multirow[t]{3}{*}{ Interdental aids } & None & 67.1 \\
\hline & Female & 56.4 & & Dental floss & 20.5 \\
\hline \multirow[t]{2}{*}{ Living area } & Urban & 80.9 & & Interdental brush & 12.4 \\
\hline & Rural & 19.1 & \multirow[t]{2}{*}{ Mouth wash } & Yes & 8.3 \\
\hline \multirow[t]{3}{*}{ Dentist visit } & None & 7.4 & & No & 91.7 \\
\hline & Regular & 11.4 & \multirow[t]{4}{*}{ Systemic diseases } & No & 88.1 \\
\hline & Irregular & 81.3 & & Hypertension & 5.7 \\
\hline \multirow{5}{*}{$\begin{array}{l}\text { Brushing } \\
\text { frequency }\end{array}$} & None & 7.4 & & Diabetes & 4.2 \\
\hline & Once a day & 50.1 & & Both & 2 \\
\hline & Twice a day & 34.4 & \multirow[t]{3}{*}{ Smoking } & No & 79.2 \\
\hline & \multirow[t]{2}{*}{$\geq 3$ a day } & \multirow[t]{2}{*}{8.1} & & Current & 14 \\
\hline & & & & Former & 6.8 \\
\hline
\end{tabular}


Table 2: Use of CPITN index to show periodontal status in different age groups.

\begin{tabular}{|c|c|c|c|c|c|c|}
\hline \multirow{2}{*}{$\begin{array}{l}\text { Age group } \\
\text { (years) }\end{array}$} & \multirow[t]{2}{*}{$\mathbf{N}$} & \multicolumn{5}{|c|}{ Percentage of persons with highest score } \\
\hline & & Healthy & Bleeding & Calculus & PPD 4-5mm & PPD $\geq 6 \mathrm{~mm}$ \\
\hline$<20$ & 122 & 0.8 & 14.8 & 68 & 15.6 & 0.8 \\
\hline $20-29$ & 674 & 0.1 & 12.6 & 67.1 & 17.4 & 2.8 \\
\hline $30-39$ & 229 & 1.7 & 6.1 & 57.6 & 27.5 & 7 \\
\hline $40-49$ & 169 & 0 & 2.4 & 59.8 & 29 & 8.9 \\
\hline 50-59 & 113 & 0 & 0 & 50.4 & 31 & 18.6 \\
\hline$\geq 60$ & 47 & 0 & 2.1 & 36.2 & 38.3 & 23.4 \\
\hline
\end{tabular}


Table 3. Multiple logistic regression estimates of the effect of various factors on the odds of a high $(\geq 3)$ CPITN score.

\begin{tabular}{|l|l|l|l|l|l|}
\hline Factor & Reference category & Odd ratio & P value & $95 \%$ CI & \\
\hline \multirow{5}{*}{ Age } & $<20$ & & & & \\
\cline { 2 - 6 } & $20-29$ & 1.2 & $\mathbf{0 . 0 1}$ & $0.5-2.6$ & \\
\cline { 2 - 6 } & $30-39$ & 1.8 & $\mathbf{0 . 0 0 1}$ & $0.8-2.8$ & \\
\cline { 2 - 6 } & $40-49$ & 3.3 & $\mathbf{0 . 0 0 0 1}$ & $1.7-6.3$ & \\
\cline { 2 - 6 } & $\geq 50$ & 3.7 & $\mathbf{0 . 0 0 0 1}$ & $1.9-7.3$ & \\
\hline Sex & Male & 6.1 & $\mathbf{0 . 0 0 0 1}$ & $3.1-12.2$ & \\
\hline Area of residence & Urban and Rural & 2.24 & $\mathbf{0 . 0 0 1}$ & $1.4-3.5$ & \\
\hline Visit to dentist & Regular and irregular & 1.1 & 0.44 & $0.7-2.3$ & \\
\hline Tooth brushing & How many times per days & 0.6 & 0.48 & $0.27-1.3$ & \\
\hline Interdental aids & Floss or tooth picks. & 1.001 & $\mathbf{0 . 0 1}$ & $1.1-2.8$ & \\
\hline Mouth wash & Yes or no & 1.01 & 0.95 & $0.9-3.2$ & \\
\hline Systemic Diseases & Diabetes, hypertension or both & 1.05 & 0.95 & $0.91-3.6$ & \\
\hline Smoking & Current & 1.8 & $\mathbf{0 . 0 0 1}$ & $0.26-4.1$ & \\
\cline { 2 - 6 } & Former & 1.4 & $\mathbf{0 . 0 1 4}$ & $1.1-1.1$ & \\
\hline Missing teeth & ??? & 0.97 & 0.14 & $0.93-1.01$ & \\
\hline PI & ??? & 0.9 & 0.84 & $0.3-2.5$ & \\
\hline BI & ??? & 0.5 & 0.24 & $0.2-1.4$ & \\
\hline
\end{tabular}

\section{Figures}




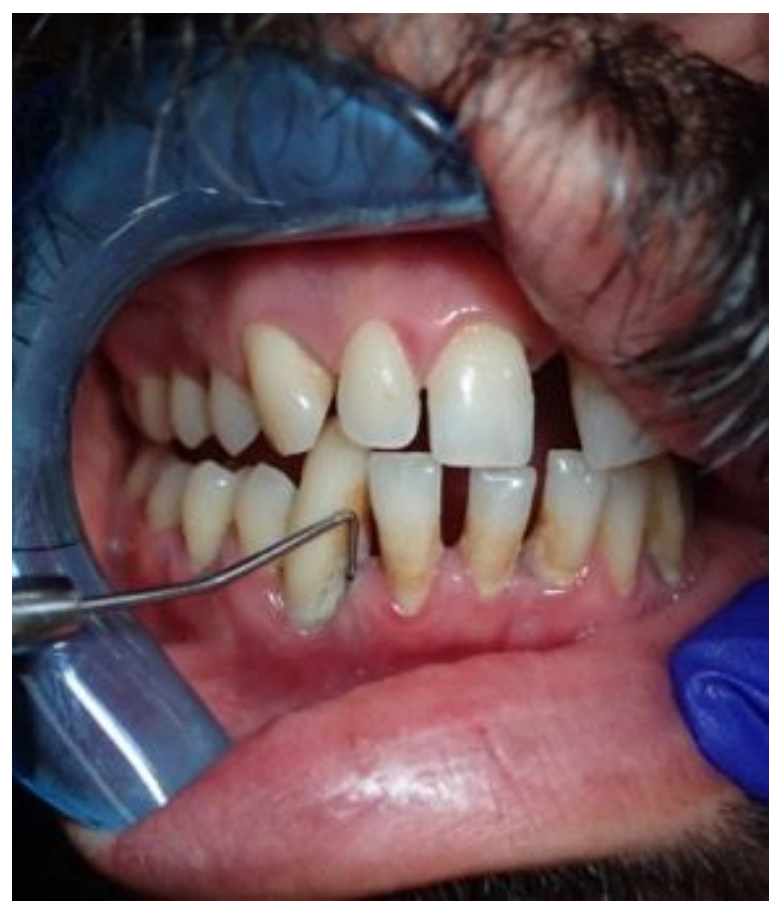

Figure 1

Periodontal examination by $\mathrm{WHO}$ probe 


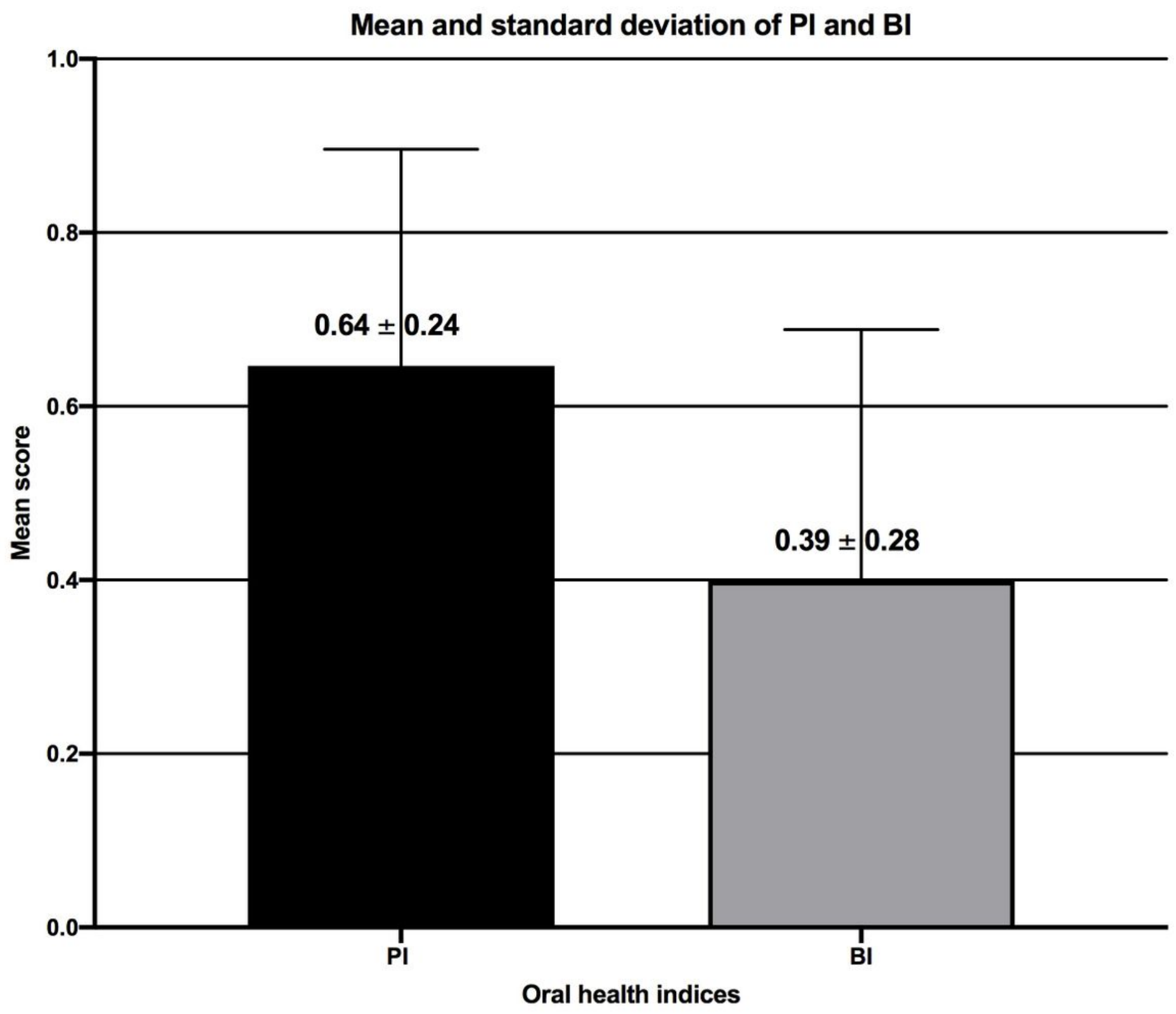

Figure 2

Mean plaque index and bleeding index of examined subjects. 


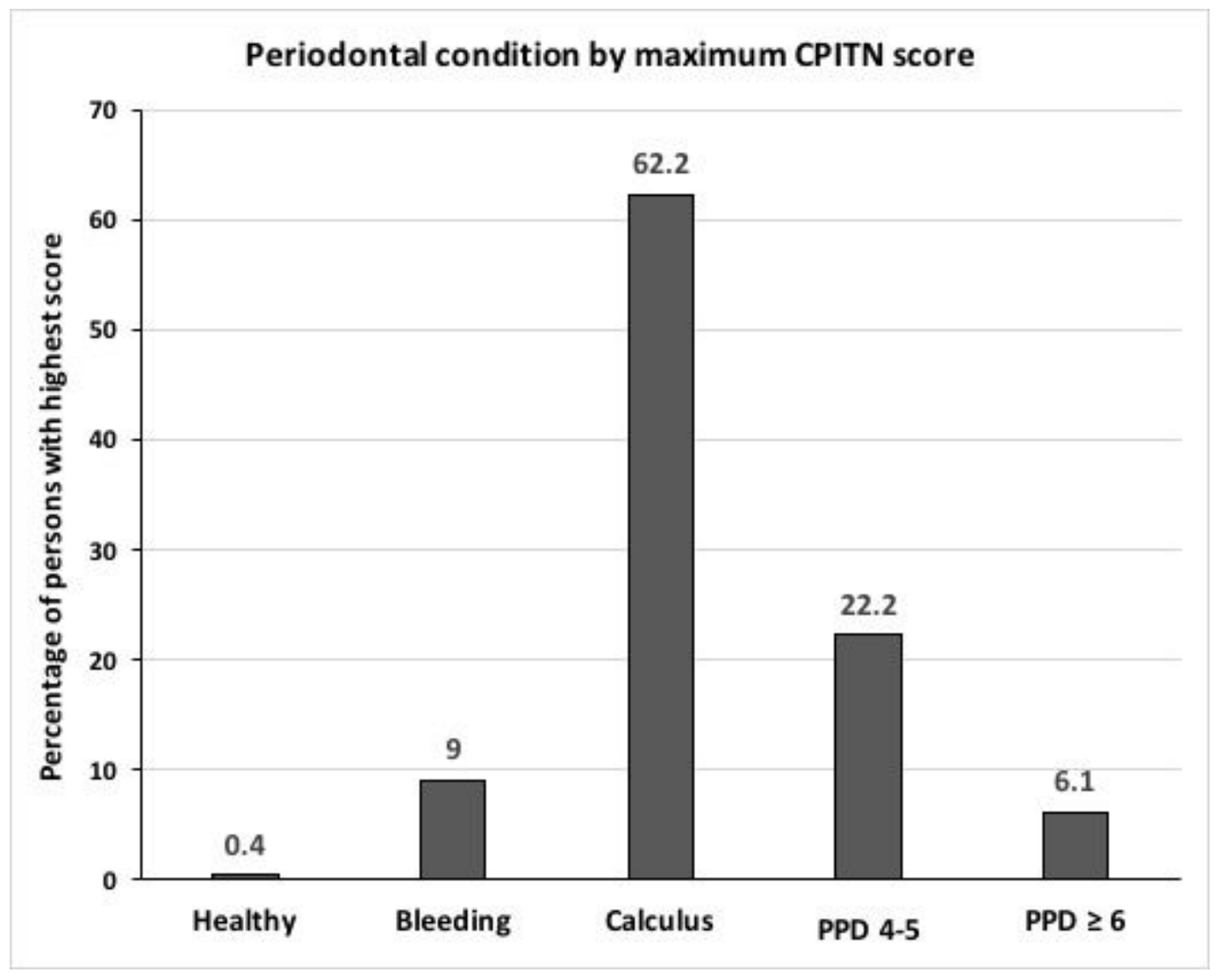

Figure 3

Periodontal condition of whole mouth using CPITN index. 


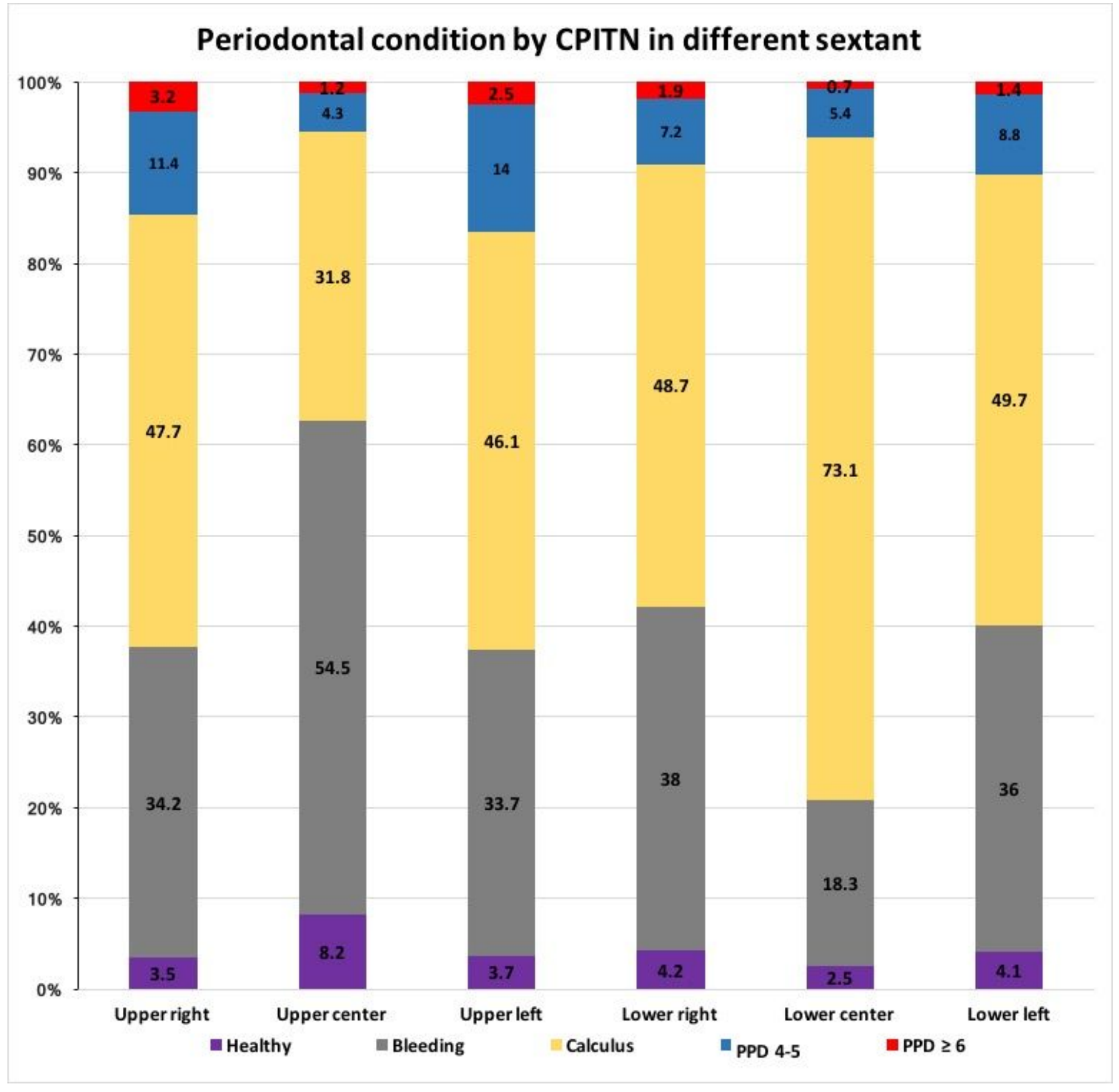

Figure 4

Periodontal condition in each sextant using CPITN index. 